\title{
Molecular Dynamics Simulations of Influence of Surface Temperature on Fluorine Etching of Silicon
}

\author{
Jianwei Wang, Yixu Song, Xiaodi Deng, Jinchun Li, and Tianling Ren
}

\begin{abstract}
Molecular dynamics simulation of the reactions between gaseous fluorine atoms and silicon are performed using the development Tersoff-Brenner potential at the temperature from $500 \mathrm{~K}$ to $1200 \mathrm{~K}$. The simulation results show that the $\mathrm{Si}$ surface temperature significantly affects the $F$ etching. For instance, as the surface temperature rises up, the numbers of $F$ atoms deposited on and scattered by $\mathrm{Si}$ surface decrease, at the same times, the number of the sputtering fluorine atoms and the reactive $F$ atoms with surface to produce volatile compounds increase. In addition, the quantity of the $\mathrm{F}$ etched $\mathrm{Si}$ atoms increased with an increase of the surface temperature.
\end{abstract}

Index Terms-Molecular dynamics, etching, temperature, silicon, fluorine.

\section{INTRODUCTION}

In recent years, plasma etching technology is widely used in the fields of microelectronics and semiconductor [1]-[3]. Over the years, people have been using halogen group $(\mathrm{F}, \mathrm{Cl}$, $\mathrm{Br})$ plasma silicon material etching ${ }^{[4-7]}$. Searching Fluorine plasma etching mechanism of $\mathrm{Si}$ for improving the etching process to improve the etching efficiency, has a great significance. Due to the complexity of the plasma environment as well as its complexity and the role of the material surface and experimental conditions limitations, which makes it difficult to fully understand the etching microscopic mechanism.

As a way to research Micro-physical properties of material, molecular dynamics(MD) simulation method has been widely used to study plasma surface interaction areas, and has made a consistent and experimental results [8]-[12]. In order to study the microscopic mechanism of the fluorine-containing plasma etching of silicon, Graves and Abrams develop the Tersoff-Brenner potential function, the description of this potential function is used to C-Si-F system.

In this paper, MD method has been used to simulate the dynamic process of low energy $\mathrm{F}$ atoms etching $\mathrm{Si}$ at different temperatures. Analyzes the impact of temperature

Manuscript received October 26, 2012; revised December 5, 2012. This work was supported by the National Science and Technology Major Project under Grant No.2011ZX2403-002.

J. Wang and T. Ren are with the Institute of Microelectronics, Tsinghua University. Beijing, China. (e-mail. jlu982309@ 126.com, RenTL@tsinghua.edu.cn)

Y. Song and X. Deng are with the State Key Laboratory on Intelligent Technology and Systems, Tsinghua National Laboratory for Information Science and Technology, Department of Computer Science and Technology, Tsinghua University, Beijing,China (e-mail. songyixu@163.com, dixiaodeng@gmail.com)

$\mathrm{J} . \mathrm{Li}$ is with the Department of Physics, University of Science and Technology, Beijing, China (e-mail. jinchunn@yahoo.com.cn) on the etching. The work on the treatment of the F plasma etching of $\mathrm{Si}$ has a certain reference value.

\section{Modeling AND Simulation MethodS}

\section{A. Simulation Method}

Theroretical work has focused on the use of ab initio calculations and molecular dynamics and melocular dynamics simulation to study reactions between both $\mathrm{F}$ atom and $F_{2}$ molecular with the $\operatorname{Si}\{100\}$ surface. A potential energy function with two-body and three-body terms to model silicon-silicon, fluorine- fluorine, and silicon-fluorine interactions was first developed by Stillinger and Weber(SW). The three-body terms are necessary to model the tetrahedral bonding of silicon. The limitation of the SW parameterization of the SW potential energy function is that it was fit to experimental gas-phase data on $\mathrm{SiF}_{\mathrm{X}}$ Species and thus, was not designed to model interactions of fluorine with a silicon sruface $\{100\}$ sruface, Weakliem, Wu, and Carter (WWC) reparameterized the SW potentiall [13] for the silicon-fluorine interactions. Abrams and Graves have developed a C-Si-F system potential and based on the Tersoff-Brenner(TB) form potential and used it to investigate CF $x(x=1-3)$ etching silicon. They also demonstrated that a $\mathrm{Si} x \mathrm{C} y \mathrm{~F} z$ reaction layer was formed and its thickness increased with the incident energy. In recent years, the SW and TB potential energy function are widely used. Therefore, in this work, the simulation of silicon-fluorine etching with the Tersoff-Brenner potential which devleped by Graves ${ }^{[14]}$ are performed. Incident fluorine atoms are used with kinetic energies ranging from $2.0 \mathrm{eV}$ to $15 \mathrm{eV}$. The reaction of the temperature is from $500 \mathrm{~K}$ to $1200 \mathrm{~K}$. The surface that $\mathrm{Si}$ target substrate consists of maximum 1728 atoms and its maximum dimention is $32.568 \AA \times 32.568 \AA \times 32.568 \AA$, and timestep is $0.1 \mathrm{fs}$. The $\mathrm{F}$ atoms injection direction is $<0 \overline{10}\rangle$.

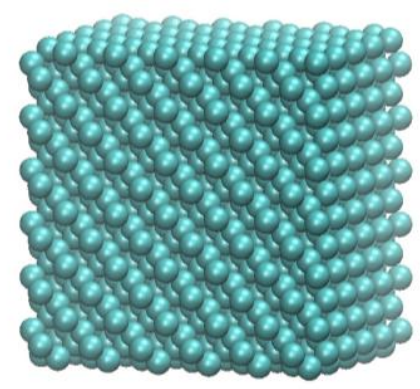

Fig. 1. The silicon model of the MD simulation

\section{B. Si-F System Potential}

In the system of the simulation, first of all, we need catch the parameters of the potential. For two elements system, 
$\mathrm{Si}-\mathrm{Si}, \mathrm{F}-\mathrm{F}$, and $\mathrm{Si}-\mathrm{F}$ potential must been considered.

We used the same function forms as Tersoff-Brenner potential. We briefly describe the functional form of the potential here. We describe each atom in a closed system by indices $i, j$, and $k$. The interatomic potential energy between two atoms $i$ and $j$ is defined as

$$
E_{i j}=V_{R}\left(r_{i j}\right)-B_{i j} V_{A}\left(r_{i j}\right)
$$

where $r_{i j}$ is the distance between atoms $i$ and $j, V_{R}$ and $V_{A}$ are the repulsive and attractive potentials, and $B_{i j}$ is an average empirical bond-order function. $V_{R}$ and $V_{A}$ are defined as

$$
V_{R}\left(r_{i j}\right)=f_{i j}\left(r_{i j}\right) \frac{D_{i j}^{(e)}}{S_{i j}-1} \exp \left[-\sqrt{2 S_{i j}} \beta_{i j}\left(r_{i j}-R_{i j}^{(e)}\right)\right]
$$

and

$$
V_{A}\left(r_{i j}\right)=f_{i j}\left(r_{i j}\right) \frac{D_{i j}^{(e)} S_{i j}}{S_{i j}-1} \exp \left[-\sqrt{2 S i_{j}} \beta_{i j}\left(r_{i j}-R_{i j}^{(e)}\right)\right]
$$

Here $S_{i j}$ is a constant, and $R_{i j}^{(e)}$ and $D_{i j}^{(e)}$ are equilibvrium bond length and bond energy when the bond order is one. Exponent constant $\beta_{i j}$ is related to the force constant of the bond as

$$
\beta_{i j}=\sqrt{k /\left(2 D_{i j}^{(e)}\right)}
$$

In order to cut off the potential smoothly, a switching function $f_{i j}$ is defined as follows.

$$
f_{i j}\left(r_{i j}\right)=\left\{\begin{array}{cc}
1 & r<R_{i j}^{(1)} \\
\left\{1+\cos \left[\pi\left(r_{i j}-R_{i j}^{(1)}\right) /\left(R_{i j}^{(2)}-R_{i j}^{(1)}\right)\right]\right\} & R_{i j}^{(1)}<r<R_{i j}^{(2)} \\
0 & r>R_{i j}^{(2)}
\end{array}\right.
$$

The total number of atoms bonded to atom $i, N_{i}^{(t)}$, the number of adjoining fluorine atoms $N_{i}^{(F)}$ is important to determine bond orders.

$$
N_{i}^{(F)}=\sum_{k \in \mathrm{F}, k \neq i, j} f_{i k}\left(r_{i k}\right)
$$

The details of the potential can refer to Ref. 14.

\section{After-Treatment}

We use the open source code LAMMPS as the main program to run the MD simulation calculation. The particle incident is continuous. To a single particle the interaction time is $1 \mathrm{ps}$, and the timestep is $0.1 \mathrm{fs}$. When binding energy between a particle and surface of the pattern is lower the threshold energy, the particle can be removed as a product. The threshold energy can expressed as follows.

$$
E_{b}=k_{B} T \ln (\tau A)
$$

$T$ is the surface temperature and $\tau$ is the time constant, $A$ is a constant. In this simulation the value of $\mathrm{A}$ and $\tau$ is $10^{12} \mathrm{~s}^{-1}$ and $1 \mu$ s. $k_{\mathrm{B}}$ is Boltzmann constant.

\section{Results AND Discussion}

In the simulation process of fluorine atoms etching the surface of silicon, verlet algorithm is used to solve Newton's equation of motion. The initial position of the incident $\mathrm{F}$ particles randomly set in the $x y$ direction, maintained in a horizontal $z$-direction in a same altitude. The injecting direction of $\mathrm{F}$ particles is need to be kept perpendicular to the ground. In the samples of $x$ and $y$ direction using periodic boundary condition and the bottom four layers atoms position need fixed on.

Fig. 2 illustrates at different times the snapshots of the simulation. From the picture we find that the more the time went on, the more the $\mathrm{F}$ atoms injected into the Si surface. And at the same time, in the surface of silicon, the F atoms that injected into the Si formed a reaction layer. The thickness of the reaction layer increase over time to reach a peak. At first $\mathrm{F}$ atoms and $\mathrm{Si}$ atoms formed $\mathrm{SiF}$, than the $\mathrm{SiF}$ and another $\mathrm{F}$ atom formed $\mathrm{SiF}_{2}, \mathrm{SiF}_{3}$, and $\mathrm{SiF}_{4}$. The $\mathrm{SiF}_{4}$ as a gas product to be removed. Then the silicon surface was etched by $\mathrm{F}$ atoms. As the $\mathrm{F}$ atoms inject constantly, the surface etched at the same time.

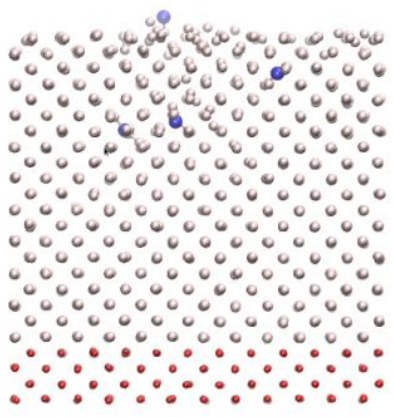

(a)

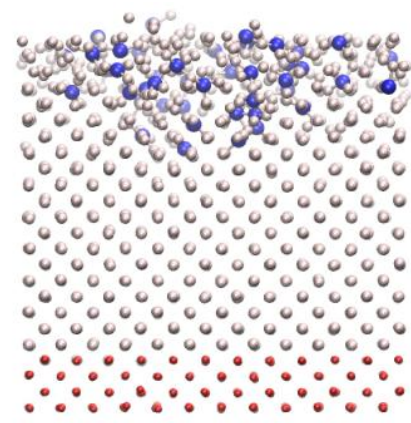

(b)

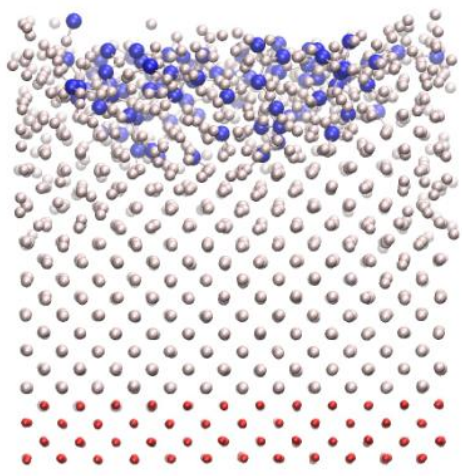

(c) 


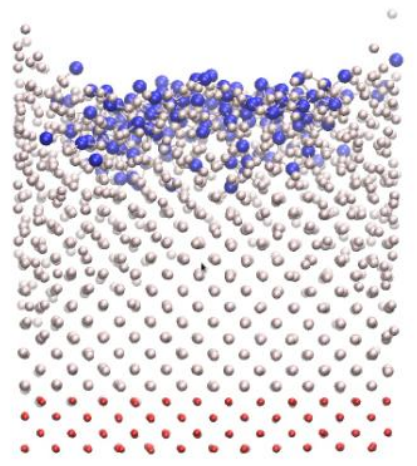

(d)

Fig. 2. The snapshots of the simulation at different times $(1 \mathrm{ps}, 50 \mathrm{ps}$, 100ps,200ps).The white balls are the silicon atoms, the blue ones are the $\mathrm{F}$ atoms. The temperature is $300 \mathrm{~K}$ and the incident atom energy is $10 \mathrm{eV}$.

Fig. 3 illustrates at different temperatures for different energy bombardment of $\mathrm{F}$ atoms is deposited on the surface of the sample after 500 times. From the figure we could find that the amount of deposition of $\mathrm{F}$ atoms, In the initial stage, increasing dramatically with the rise of time. The deposited amount of $\mathrm{F}$ atoms on the surface has a direct relationship with temperature of the sample sruface. The deposition amount of $\mathrm{F}$ atoms is reducing with rise of temperature. Comparison for the $0.5 \mathrm{eV}$ and $10 \mathrm{eV}$, we found that the deposited amount of $\mathrm{F}$ atoms increases with energy increasing. Deposited on the surface of the sample, scattering and after deposition of the sputtering may occured later in the injected $\mathrm{F}$ atom, and the sample surface atomic. Only less than 10 percent injected $\mathrm{F}$ atoms will deposit on the surface of silicon.
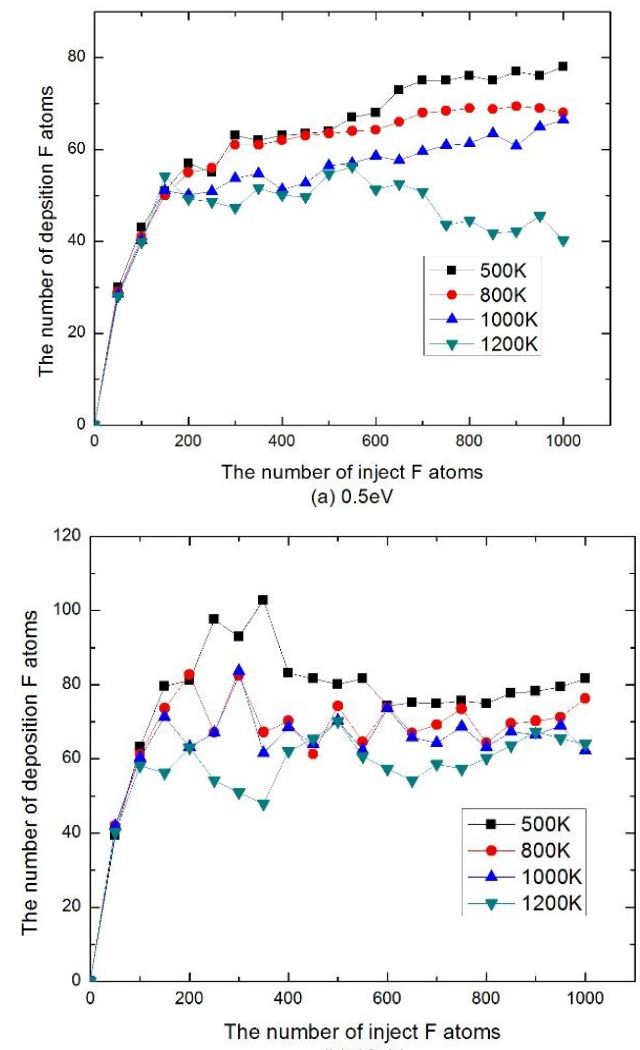

(b) $10 \mathrm{eV}$

Fig. 3. The number of deposition $\mathrm{F}$ atoms as a function of the number of inject $\mathrm{F}$ atoms.
Fig. 4 shows when the etching yield of $\mathrm{Si}$ atoms in the sample of $\mathrm{Si}$, as a function of the number of injected $\mathrm{F}$ atoms at different temperatures. From the picture we can find that in the two energy condition, the etching yield of $\mathrm{Si}$ is increasing with the rise of temperature. The Fig. 4 (a) shows that when the the injected $\mathrm{F}$ Atomic energy is $0.5 \mathrm{eV}$ and the temperature is $500 \mathrm{~K}$ or $800 \mathrm{~K}$, etching product does not appeared. But when the temperature is beyonged $1000 \mathrm{~K}$ the etching obviously ocurred. The Fig. 4 (b) showed that if energy of $\mathrm{F}$ is $10 \mathrm{eV}, \mathrm{F}$ atoms etching occurred when the temperature is $500 \mathrm{~K}$ or beyonged it.

From the two picture we also find that if the incident energy is identical. The etch rate (ratio of the number of etched silicon to the number of incident fluorine atoms) is as high as temperature. And when the temperature is same, the more the energy the more the etch rate.

This result has been confirmed in the experiment [15].

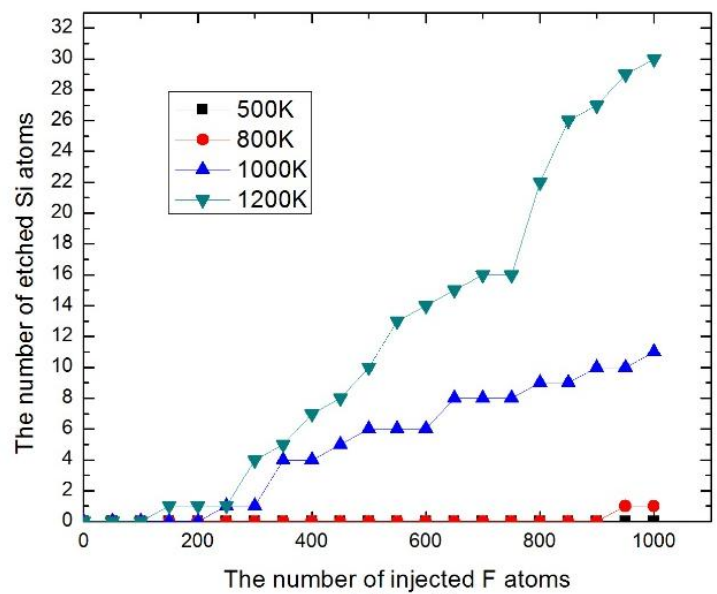

(a) $0.5 \mathrm{eV}$

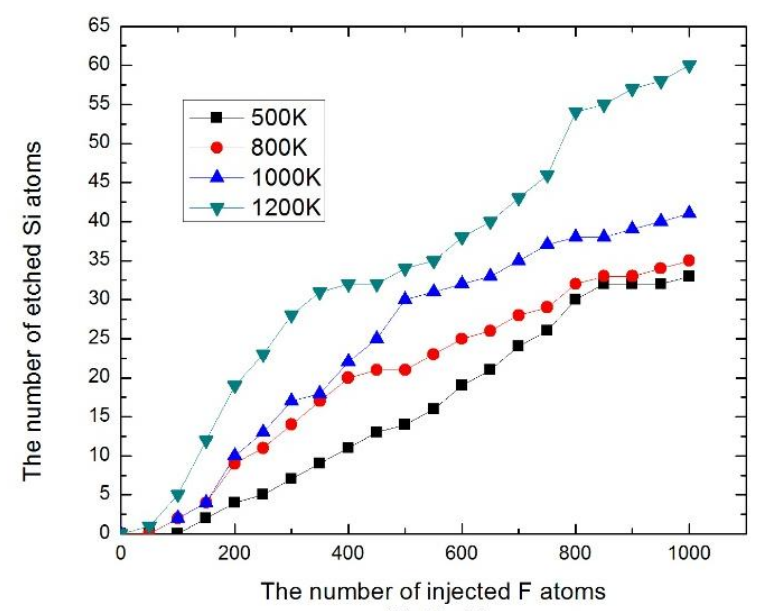

(b) $10 \mathrm{eV}$

Fig. 4. The number of etching yield $\mathrm{F}$ atoms as a function of the number of inject $\mathrm{F}$ atoms.

\section{CONCLUSION}

For micro-scale MD simulations of fluorocarbon systems, as the surface temperature rises up, the numbers of $\mathrm{F}$ atoms deposited on and scattered by Si surface decrease, at the same times, the number of the sputtering fluorine atoms and the reactive $\mathrm{F}$ atoms with surface to produce volatile compounds 
increase. In addition, the quantity of the $\mathrm{F}$ etched $\mathrm{Si}$ atoms increased with an increase of the surface temperature.

\section{REFERENCES}

[1] J. P. Chang and J. W. Cobum, "Plasma-surface interactions," Journal of Vacuum Science \& Technology A, 2003, pp. S145-S151.

[2] Z. L. Dai, M Mao, and Y. N. Wang, "The physics of plasma etching," Physics, 2006, pp. 693-698.

[3] H. Jansen, H. Gardeniers, M. D. Boer, M. Elwenspoek, and J. Fluitman, "A survey on the reactive ion etching of silicon in microtechnology," Journal of Micromechanics and Microengineering, 1996, pp. 14-28.

[4] C. M. Aldao, A. Agrawal, R. E. Butera, and J. H. Weaver, "Atomic processes during $\mathrm{Cl}$ supersaturation etching of $\mathrm{Si}(100)-(2 \times 1)$," Physical Review B, 2009, pp. 125303(1-5).

[5] D. J. Lu and Z. D. Jiang, "Plasma Cyro-etching of High Aspect Ratio Silicon Crystal Structures," Chinese Journal of Vacuum Science and Technology, 2007, pp. 25-30.

[6] S. J. Samukawa, B Jinnai, F. Oda, and Y. Morimoto, "Surface Reaction Enhancement by UV irradiation during Si Etching Process with Chlorine Atom Beam," Japanese Journal of Applied Physics, 2007, pp. L64-L66.

[7] F. J. Gou, M. A. Gleeson, and A. W. Kleyn, "Theoretical modeling of energy redistribution and stereodynamics in $\mathrm{CF}$ scattering from $\mathrm{Si}(100)$ under grazing incidence," Phys Chem Chem Phys, 2006, pp. $5522-5534$.

[8] H. Ohta and S. Hamaguchi, "Classical interatomic potentials for $\mathrm{Si}-\mathrm{O}-\mathrm{F}$ and $\mathrm{Si}-\mathrm{O}-\mathrm{Cl}$ systems," Journal of Chemical Physics, 2001, pp. 6679-6690.

[9] D. E. Hanson, J. D. Kress, and A. F. Voter, "An interatomic potential for reactive ion etching of Si by $\mathrm{Cl}$ ions," Journal of Chemical Physics, 1999, pp. 5983-5988.

[10] H. David and D. B. Graves, "Atomistic simulations of spontaneous etching of silicon by fluorine and chlorine," Journal of Applied Physics, 2004, pp. 791-798.
[11] H. Ohta and S. Hamaguchi, "Molecular dynamics simulation of silicon and silicon dioxide etching by energetic halogen beams," Journal of Vacuum Science \& Technology A, 2001, pp. 2373-2381.

[12] D. E. Hanson, A. F. Voter, and J. D. Kress, "Molecular dynamics simulation of reactive ion etching of $\mathrm{Si}$ by energetic $\mathrm{Cl}$ ions," Journal of Applied Physics, 1997, pp. 3552 -3559.

[13] A. T. Weber, H. Frank, and Stillinger, "Dynamical branching during fluorination of the dimerized $\mathrm{Si}(100)$ surface. A molecular dynamics study," Journal of Chemical Physics, 1990, pp. 6239-6245.

[14] C. F. Abrams and D. B. Graves, "Molecular dynamics simulations of Si etching by energetic $\mathrm{CF}_{3}{ }^{+}$," Journal of Applied Physics, 1999, pp. 5938-5948.

[15] H. F. Winters, "Etch products from the reaction of $\mathrm{XeF} 2$ with $\mathrm{SiO}$, $\mathrm{Si} 3 \mathrm{~N} 4, \mathrm{SiC}$, and $\mathrm{Si}$ in the presence of ion bombardment," Journal of Vacuum Science \& Technology B, 1983, pp. 927-931.

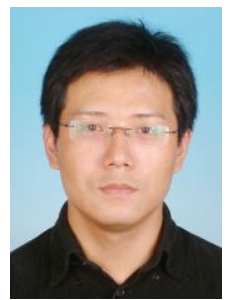

Jianwei Wang is working as an associate researcher in Institute of Microelectronics, Tsinghua University. Beijing, China. He received his $\mathrm{PhD}$ in engineering mechanics from University of Science and Technology,Beijing(USTB) . His research areas are on plasma physics etching and deposit and radiation damage computer simulation. 